\title{
Online process monitoring of a batch distillation by medium field NMR spectroscopy
}

\author{
Anne Friebel, Erik von Harbou*, Kerstin Münnemann, Hans Hasse \\ Laboratory of Engineering Thermodynamics, University of Kaiserslautern, Germany
}

\begin{abstract}
Medium field NMR spectrometers are attractive for online process monitoring. Therefore, in the present work, a single-stage laboratory batch distillation still was coupled online with a medium field NMR spectrometer. This enables quantitative non-invasive measurements without calibration. The technique was used for studying isobaric and isothermal residue curves in two ternary systems: (dimethyl sulfoxide + acetonitrile + ethyl formate) and (ethyl acetate + acetone + diethyl ether) and boiling curves and high-boiling azeotropes in two binary systems: (acetic acid + pyridine) and (methanol + diethylamine). The results of the online NMR spectroscopic analysis were compared to results from offline analysis as well as to results from thermodynamic modeling using NRTL parameters that were parametrized with literature data. The new method for online process monitoring gives reliable results and is well-suited for fast and robust measurements of residue curves. Keywords: medium field NMR spectroscopy, Benchtop NMR spectrometer, online analysis with flow NMR, single-stage batch
\end{abstract}

\footnotetext{
*Corresponding author: Erik von Harbou (erik.vonharbou@mv.uni-kl.de), presently with BASF, SE, reaction technology
} 
distillation, residue curve, high-boiling azeotrope

\section{Introduction}

Online process monitoring is important for process control and optimiza3 tion. Recently, robust and small medium field nuclear magnetic resonance 4 (NMR) spectrometers have become available. These instruments use perma-

nent magnets but have a field that is high enough to yield a resolution that enables to distinguish component peaks in the spectrum, which is the prerequisite for quantitative spectroscopy. Medium field devices are particularly attractive for online process monitoring because, in contrast to conventional high field instruments, they do not require cryogenic media, climatized and vibration-free installation, and they are comparatively cheap. Despite the lower sensitivity and chemical resolution compared to high field NMR spectrometers, the spectra obtained by medium field spectroscopy can be evaluated quantitatively. The quantification is possible without calibration, which is an advantage over other spectroscopic methods e.g. IR spectroscopy. Furthermore, due to the high dispersion of NMR spectroscopy, peak overlaps are often no problem, and, if they occur, advanced techniques are available that enable a reliable quantification also in these cases $[1,2]$. Because NMR spectroscopy is applicable to flowing liquid samples, the spectrometer can be coupled to the process by a sample loop. This allows a non-invasive measurement with high temporal resolution where pressure, temperature, and the composition of the mixture is not affected by the analysis.

A variety of applications of high field NMR spectroscopy for online process monitoring have been described in the literature [3-6], but they are limited 
to laboratory conditions. The advent of commercially available medium field NMR spectrometers opened up new possibilities. Various publications describe applications of medium field NMR spectrometers for monitoring of reactions [7-11] and processes [12-16] and for controlling the product quality during production [17-20]. To our knowledge, however, NMR spectroscopy (neither with medium nor with high magnetic fields) has not been used yet for online monitoring of fluid separation processes such as distillation. Therefore, in the present work, a single-stage batch distillation was coupled online with a medium field NMR spectrometer. This enables, e.g. determining residue curves and high-boiling azeotropes. More generally, $p T x$-data of vapor-liquid equilibria (VLE) can be determined, where $p$ is the pressure, $T$ is the temperature and $x$ the liquid-phase composition. This is possible for systems with a many of components.

The setup that was used in the present work consists of a simple batch distillation still which was coupled online with a sample loop to a medium field NMR spectrometer. The sample is taken from the liquid phase and the NMR spectrometer is operated in flow-mode. The control is designed such that the distillation can be carried out at isobaric as well as at isothermal conditions. In preliminary investigations the setup was characterized and the residence time in the sample loop as well as the maximal volume flow rate for sufficient magnetization were determined [21, 22]. The setup was then used for studying isobaric and isothermal residue curves in two ternary test systems: (dimethyl sulfoxide (DMSO) + acetonitrile $(\mathrm{ACN})+$ ethyl formate $(\mathrm{EF}))$ and (ethyl acetate $(\mathrm{EA})+$ acetone $(\mathrm{ACT})+$ diethyl ether $(\mathrm{DE}))$. It was also applied for measuring the isobaric boiling curve and for determining the 
high-boiling azeotrope in two binary systems: (acetic acid (AA) + pyridine $(\mathrm{P})$ ) and (methanol (M) + diethylamine (DEA)). For comparison, the system $(\mathrm{DMSO}+\mathrm{ACN}+\mathrm{EF})$ was investigated by offline gas chromatography $(\mathrm{GC})$ analysis as well. The experimental data from this work were compared to results that were obtained from thermodynamic modeling, based on NRTL, with model parameters that were determined from experimental data from the literature.

\section{Experimental section}

\subsection{Chemicals}

Table 1 lists the chemicals that were used in the present work. All chemicals were used without further purification. No side components were detected in the sample analysis. Figure 1 shows the chemical structures of all components that were quantified by NMR spectroscopy together with the nomenclature that is used for the peak assignment.

\subsection{Experimental setup}

Figure 2 shows the distillation setup. It consists of an electrically heated glass batch distillation still (total volume $500 \mathrm{ml}$ ) that is connected to a medium field NMR spectrometer by a sample loop in which the liquid phase circulates. The setup can be operated at isobaric or at isothermal conditions. In case of the isothermal measurement the pressure was manipulated in a control loop to maintain the temperature in the still.

The liquid in the distillation still (about $210 \mathrm{ml}$ at the beginning) was continuously stirred during the experiment (RCT Basic with flask carrier, 
IKA). The changing magnetic field of the magnetic stirrer was shielded with a $\mu$-metal foil. The ascending vapor was condensed in a water cooled Liebig condenser and was collected in the distillate flask.

The temperature of the liquid phase was measured with a calibrated PT100 thermometer connected to a multimeter (series 2700 Multimeter, Keithley Instruments, accuracy: $\pm 0.1^{\circ} \mathrm{C}$ ). The pressure was measured with piezo sensors (VSR53DL and VSC42MA4, Thyracont Vacuum Instruments, accuracy: $\pm 0.3 \%$ ). Temperature and pressure were continuously recorded with the software LabView. The control system that was realized using LabView enables both isobaric and isothermal operation. In case of the isothermal measurement the pressure was adjusted in a control loop to maintain constant temperature in the still.

The sample loop was realized with a PEEK capillary $\left(d_{\mathrm{i}}=1 \mathrm{~mm}\right)$, which was passed through the medium field NMR spectrometer $(42.5 \mathrm{MHz}$, Spinsolve Carbon, Magritek). The flow was maintained by a double piston highpressure pump with damping piston (WADose LITE HP, Flusys, accuracy: $\pm 0.01 \mathrm{ml} / \mathrm{min}$ ) and was measured with a Coriolis flow sensor (Mini CoriFlow, Bronkhorst, accuracy: $\pm 0.2 \%$ ). The flow sensor's signal was used to control the pump. This set-up gave very good results and we would have liked to keep it also for the isothermal measurements. However, in these measurements, the pressures were sometimes so low that the Flusys pumps did not work properly. Therefore, in the isothermal measurements a high precision dosing pump (HPD3351, Bischhoff Chromatography, accuracy: $\pm 2 \%$ ) was used. To reduce pulsation, the flow was split. This pump gave acceptable results but is not considered as an optimal system for the present application. 
Discontinuities of the flow lead to a larger scattering of the analytical results. Volume and mass flow in the sample loop were continuously recorded with the software LabView.

\subsection{Procedure}

All chemicals were degassed before the experiment. The liquid feed mixtures were prepared gravimetrically using an analytical balance (XS603S DeltaRange, Mettler Toledo, accuracy: $\pm 10 \mathrm{mg})$. Isobaric experiments were performed at 970 mbar, isothermal experiments were performed at $303 \mathrm{~K}$ and $323 \mathrm{~K}$. The flow rate in the sample loop was set to $\dot{V}=0.2 \mathrm{ml} / \mathrm{min}$. It was shown in preliminary experiments that this flow rate is sufficient to ensure complete magnetization of the components prior to entering the active volume of the NMR spectrometer. More information on this is given in the Supplementary Material. ${ }^{1} \mathrm{H}$ NMR spectra were recorded with intervals of $1 \mathrm{~min}$. As the sample loop was not thermostated, the circulating liquid cooled down during analysis. The small reflux from the sample loop has no significant influence on the temperature of the liquid in the still. For a comparative offline analysis, a syringe was used to withdraw samples $(0.5 \mathrm{ml})$ from the liquid phase in the still.

Because the fluid needs to be transported from the still to the active volume of the NMR spectrometer, where the analysis takes place, there is a time delay between the measurement of temperature and pressure and the measurement of the composition. This was determined by residence time distribution measurements. The delay was found to be $455 \mathrm{~s}$ in the isobaric setup and $209 \mathrm{~s}$ in the isothermal setup. By taking this time delay into account each measured composition was assigned to the temperature and pressure in 
the still. When we report concentrations in the still as a function of time, the time refers always to the time at which the sample was withdrawn from the still. It is calculated from the time of the analysis by substracting the time delay for transferring the sample to the NMR spectrometer. Details on the residence time distribution measurements are reported in the Supplementary Material.

\subsection{Analysis}

The composition of the liquid phase was analyzed online by medium field NMR spectroscopy. The composition of the liquid samples that were taken from the still in the studies of the system DMSO + ACN + EF were determined by GC.

The online NMR analysis was carried out with a medium field NMR spectrometer (Spinsolve Carbon, Magritek) with a field strength of $1 \mathrm{~T}$ corresponding to a Larmor frequency of $42.5 \mathrm{MHz}$ for ${ }^{1} \mathrm{H}$. The optimization of the magnetic field homogeneity (shimming) was performed with water in the capillary using the standard procedure of the manufacturer. The ${ }^{1} \mathrm{H}$ NMR spectra were measured with the following parameters: acquisition time: $3.2 \mathrm{~s}$, $16 \mathrm{k}$ data points, one scan, $90^{\circ}$ excitation pulse. Prior to integration, post processing (baseline and phase correction) of the obtained NMR spectra was carried out with the SINC method [23]. Because of fully overlapping peaks in the system EA $+\mathrm{ACT}+\mathrm{DE}$, an indirect hard modeling (IHM) approach was used to determine the peak areas of all components in this mixture using the software PEAXACT S-Pact. For all other systems the MNova software (MestReLabs) was used for integration.

Figure 3 shows examples of ${ }^{1} \mathrm{H}$ NMR spectra for all studied systems. 
The mole fractions of the different components in the sample were calculated from the normalized peak area fractions of the corresponding peaks in the NMR spectrum. For each system, three reference samples were prepared gravimetrically to test the accuracy of the NMR analysis and to assign the peaks in the spectrum. The absolute error of the method for the reference samples was on average $0.006 \mathrm{~mol} / \mathrm{mol}$. Taking the reduced signal to noise ratio of a flowing sample into account the absolute error of the NMR method is $0.01 \mathrm{~mol} / \mathrm{mol}$. More information is given in the Supplementary Material. The offline GC analysis was carried out with an Agilent gas chromatograph with a flame ionization detector (FID) (7890A, Agilent Technologies). 1,4-dioxane was used as internal standard. Each sample was analyzed three times and the results were averaged. The absolute error of the method for the reference samples was below $0.006 \mathrm{~mol} / \mathrm{mol}$. As the composition of the reference samples is representative for the compositions investigated in the distillation experiments, the absolute error of the GC method is $0.006 \mathrm{~mol} / \mathrm{mol}$. More details on the GC method and calibration are given in the Supplementary Material.

\subsection{Modeling and simulation}

The batch distillation still was modeled as an equilibrium stage, i.e. it was assumed that the gas stream, that leaves the still is in equilibrium with the remaining liquid residue. The residue curves were obtained by solving the Rayleigh equation:

$$
\frac{\mathrm{d} x_{i}}{\mathrm{~d} \theta}=x_{i}-y_{i}
$$

where $x_{i}$ and $y_{i}$ are the mole fractions of component $i$ in the liquid and gas phase respectively and $\theta$ is a dimensionless time parameter. The vapor-liquid 
equilibrium was calculated from:

$$
p_{i}^{\mathrm{s}} \cdot x_{i} \cdot \gamma_{i}=p \cdot y_{i}
$$

where $p_{i}^{\mathrm{s}}$ is the vapor pressure of component $i$ and $\gamma_{i}$ is the liquid phase activity coefficient, which was calculated here using the NRTL model [24]. The NRTL parameters were determined as follows: for the system $\mathrm{EA}+\mathrm{ACT}+\mathrm{DE}$, the parameters were adapted from the Aspen data base as the model predictions were found to agree well with experimental literature data from the Dortmund Data Bank (DDB). For all other systems, the NRTL parameters were fitted to experimental literature data from the DDB. The vapor pressure correlations and NRTL parameters are given in the Supplementary Material.

\section{Results and discussion}

In this section, the results of the distillation experiments are presented. For the ternary systems $(\mathrm{DMSO}+\mathrm{ACN}+\mathrm{EF})$ and $(\mathrm{EA}+\mathrm{ACT}+\mathrm{DE})$ residue curves were measured in isobaric as well as in isothermal experiments (Figure 4 and Figure 5). For the binary systems $(\mathrm{AA}+\mathrm{P})$ and $(\mathrm{M}+\mathrm{DEA})$ the boiling curve and the high-boiling azeotrope were determined in isobaric experiments (Figure 6 and Figure 7). The numerical experimental online and offline data of the residue curves, the boiling curves and the high-boiling azeotropes are reported in the Supplementary Material.

\subsection{System: Dimethyl sulfoxide + Acetonitrile + Ethyl formate}

Figure 4 shows the residue curves of the distillation experiments of the ternary system DMSO + ACN + EF. Two feed compositions were used in isobaric measurements at 970 mbar as well as in isothermal measurements at 
$323 \mathrm{~K}$. As expected, the component with the highest boiling point (dimethyl sulfoxide) is enriched in the liquid phase during experiment. For the isobaric measurement experimental online NMR data is compared to experimental offline GC data. For both feeds, the NMR data is in fair agreement with the GC data. There are some systematic deviations, but they rarely exceed the cumulated analytical uncertainties of both methods. The deviations are caused by imperfections of the setup (deviations in flow rate and in pressure (isobaric measurement) and temperature (isothermal measurement)) and uncertainties in the evaluation of NMR data. This comparison proves the applicability of the online NMR setup for the monitoring of residue curves. The NRTL model predicts the experimental data well, although not perfectly. The scattering of the NMR data in the isothermal experiment is larger than that of the isobaric experiment. This is due to the different pumps that were used in the sample loop and the different control strategy, see section 2.2. The comparison shows that the isothermal set-up leaves room for improvements. There is no good reason why the scattering in the isothermal experiments could not be as low as that in the isobaric experiments, had a better pump been available. Hence, for future isothermal experiments, pumps should be used that enable maintaining constant flow rates also at low pressures. Nevertheless, the results show the general usefulness of the method.

As an alternative to the presentation of the residue curves shown in Figure 4 , also plots of the concentration of the liquid as a function of the temperature (isobaric measurement) or the pressure (isothermal measurement) can be used. Such plots are presented in the Supplementary Material. Again, 
good agreement is observed.

\subsection{System: Ethyl acetate + Acetone + Diethyl ether}

Figure 5 shows the residue curves of the distillation experiments of the ternary system EA $+\mathrm{ACT}+\mathrm{DE}$. Two feed compositions were used in isobaric measurements at 970 mbar as well as in isothermal measurements at $303 \mathrm{~K}$. As expected, the component with the highest boiling point (ethyl acetate) is enriched in the liquid phase during experiment. No offline samples were taken because of the high volatility of the components which leads to biased sample compositions. Experimental online NMR data is compared to predictions with the NRTL model. For both feeds the NMR data agrees well with the model. Again, the scattering of the NMR data in the isothermal measurement is much higher than that of the isobaric data, for the reasons discussed above.

Plots of the concentration of the liquid as a function of the temperature (isobaric measurement) or the pressure (isothermal measurement) are presented in the Supplementary Material. Again, good agreement is observed.

\subsection{System: Acetic acid + Pyridine}

Figure 6 shows the results of the distillation experiments of the binary system AA $+\mathrm{P}$, which has an high-boiling azeotrope. Two feed compositions were used in isobaric measurements at 970 mbar to measure the boiling curve and the azeotropic point of the binary system. In Figure 6a) the boiling curve is plotted as a function of mole fraction of pyridine in the liquid phase. The experimental online NMR data is compared to the NRTL model prediction. Both NMR data sets agree well with the model data. After 
the heating up (points under the boiling curve), the boiling starts at slightly lower temperatures than predicted. This results from the fact, that right after the boiling sets in, there is still nitrogen in the gas phase of the still. At constant temperature, the presence of nitrogen would lead to an increased pressure. Vice versa, for constant pressure, as in the experiment shown in Figure 6 , the presence of nitrogen leads to temperatures that are too low. The effect is present until all nitrogen is purged from the gas phase by the vaporized components.

Figures $6 \mathrm{~b}$ ) and c) show the experimental values of temperature and mole fraction of pyridine in the liquid phase as a function of time. By means of the online measurement a continuous monitoring of temperature and liquid phase composition is possible. The azeotropic point found in this work is in good agreement with the predicted one and with those published in literature, see Table 2. This comparison shows that the setup is well suited to investigate boiling curves and high-boiling azeotropes.

\subsection{System: Methanol + Diethylamine}

Figure 7 shows the results of the distillation experiments of the binary system M + DEA, which has an high-boiling azeotrope. Two feed compositions were used in isobaric measurements at 970 mbar to measure the boiling curve and the azeotropic point of the binary system. The representation of the results is the same as in Figure 6. The experimental online NMR data is well predicted by the NRTL model. As explained above, a kinetic effect occurs at the beginning of the boiling, which causes a slight discrepancy between experimental data and prediction. The azeotropic point measured with online NMR spectroscopy agrees well with the NRTL model prediction 
and with data from literature, see Table 2.

\section{Conclusions}

In the present work, a laboratory batch distillation still was coupled online with a medium field NMR spectrometer and was used for measuring residue curves, boiling curves, and high-boiling azeotropes. The liquid phase is continuously withdrawn from the still and circulates in the sample loop in which it is analyzed with the online NMR spectrometer. The sample loop is a simple PEEK capillary that passes through the spectrometer's bore. Isobaric and isothermal measurements were performed. The results from isothermal experiments scatter more than those from the isobaric measurements, as a consequence of a less favorable pump that had to be used in the sample loop. The setup was tested using two ternary zeotropic and two binary azeotropic mixtures. The residue curves obtained with the new setup were found to agree well with offline GC sampling. All experimental results were in good agreement with the predictions from a thermodynamic model that was parametrized using literature VLE data of the studied systems. The online NMR analysis of the liquid phase enables the determination of residue curves and boiling curves with high resolution. The investigation of high-boiling azeotropes is also feasible.

As the NMR measurement is non-invasive, the analysis in the sample loop does not affect the thermodynamic conditions of the system (temperature, pressure, composition of phases). This enables a simple analysis of systems with volatile components, unstable intermediates and at pressure below atmospheric pressure. The compact and robust medium field NMR spectrom- 
eters enable online monitoring of processes not only in the laboratory but also in pilot and production plants. The presented setup is simple and robust and extends the standard techniques for thermodynamic measurements. The easy access to residue curve data in multicomponent systems, that is provided by the present set-up, is particularly interesting for the validation of VLE models that were parametrized based on binary data.

\section{Acknowledgment}

The authors thank the German Research Foundation (DFG) for the financial support within the Collaborative Research Center SFB/TRR 173 Spin $+X$. The authors thank Johnnie Phuong and Felix Selzer for their contribution to the experiments of this work. 

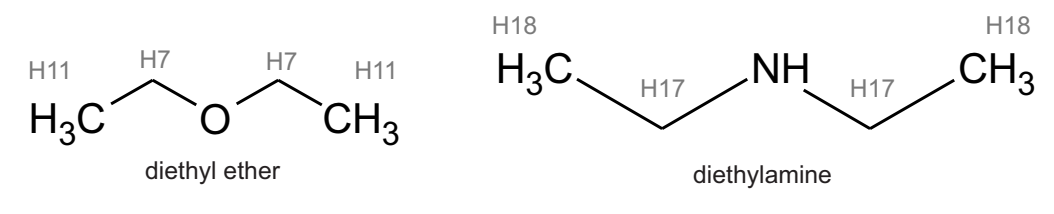

$\overbrace{\text { ethyl acetate }}^{\mathrm{CH}_{3}}$<smiles>CCOC=O</smiles><smiles>CO</smiles><smiles>c1ccncc1</smiles><smiles>CC(C)=O</smiles><smiles>CC(=O)O</smiles><smiles>CC#N</smiles>

Figure 1: Chemical structures of the components that were analyzed by ${ }^{1} \mathrm{H}$ NMR spectroscopy and nomenclature used for the peak assignment.

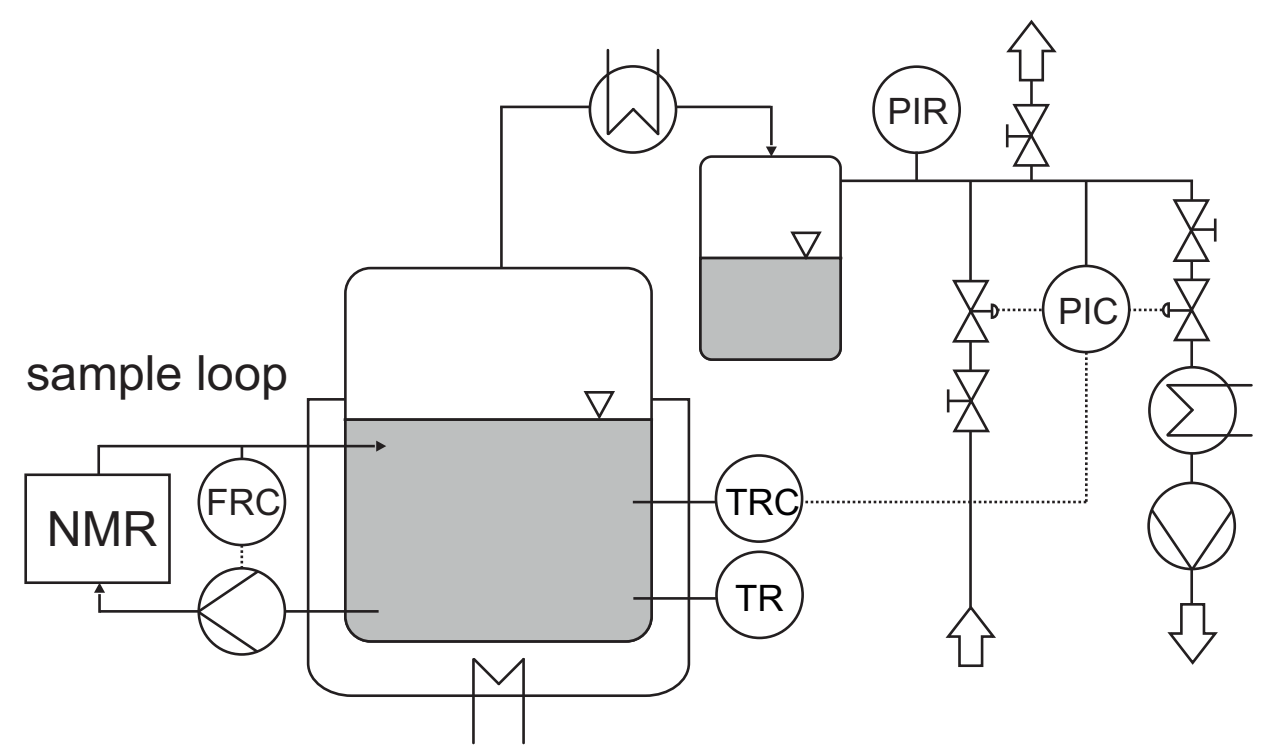

Figure 2: Experimental setup for the batch distillation with online NMR analysis. 


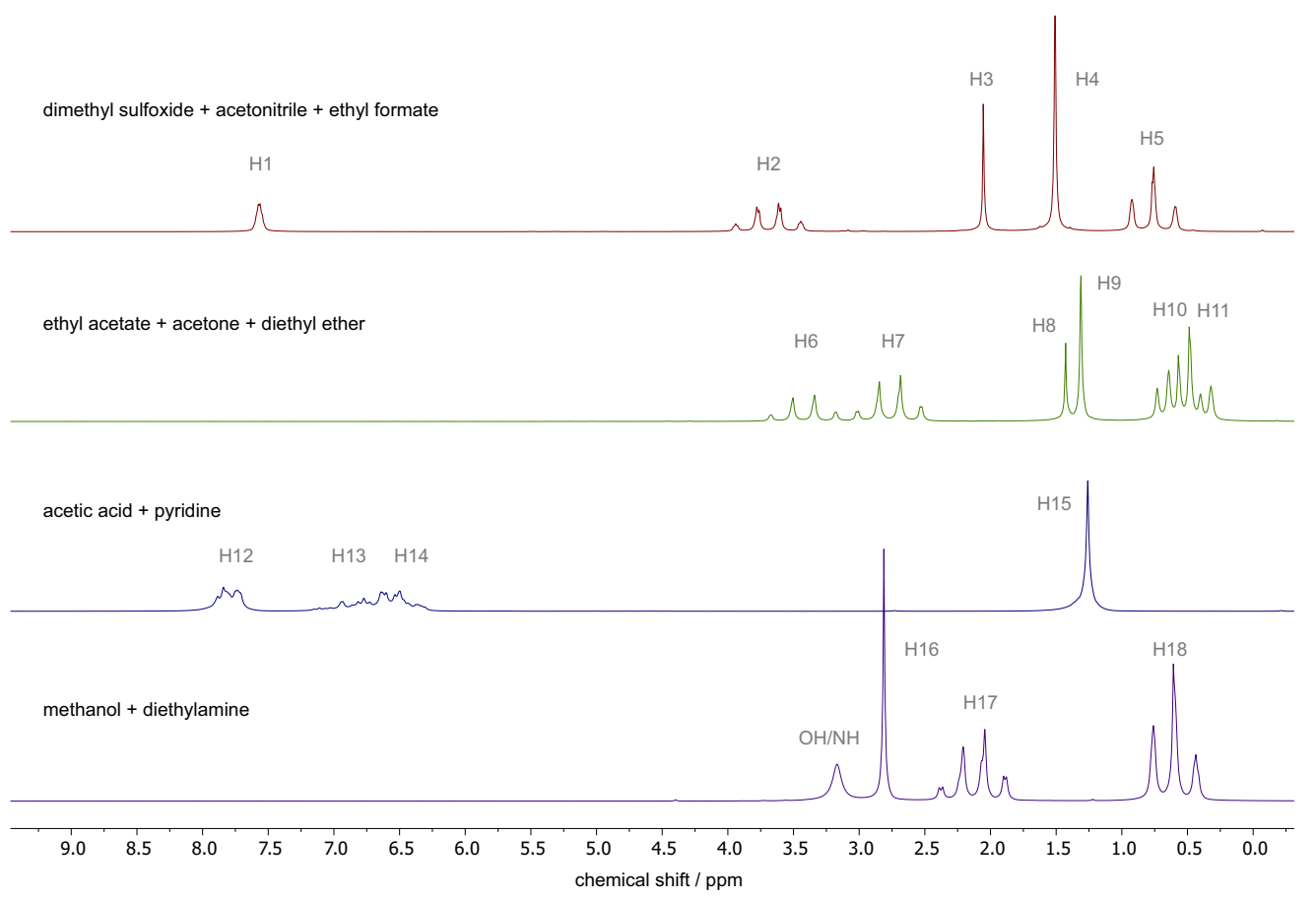

Figure 3: ${ }^{1} \mathrm{H}$ medium field NMR spectra of reference samples of the investigated systems. Peak assignment, see Figure 1. 

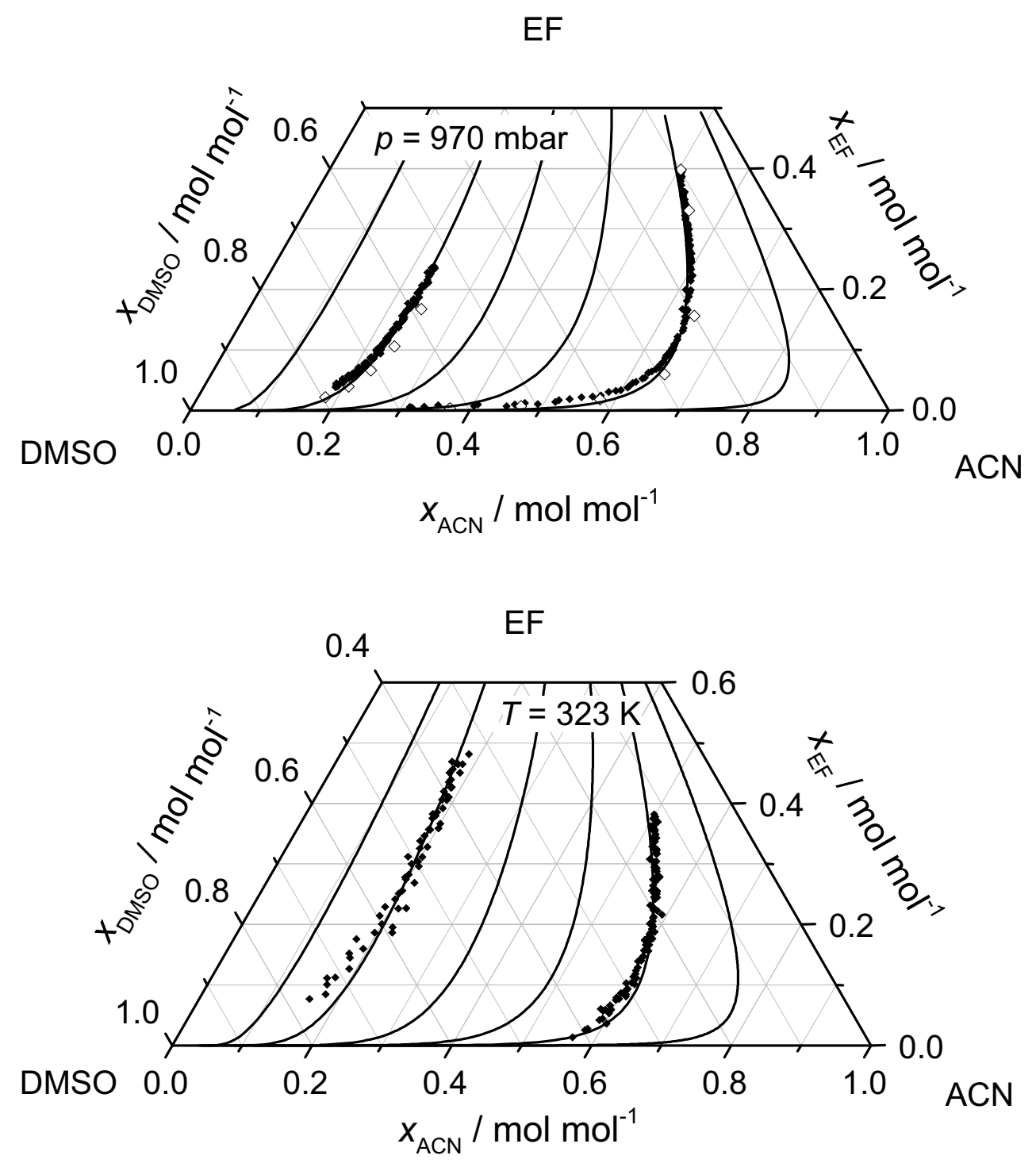

Figure 4: Results from measurements of two isobaric $(p=970 \mathrm{mbar})$ and two isothermal $(T=323 \mathrm{~K})$ residue curves in the system dimethyl sulfoxide (DMSO) + acetonitrile $(\mathrm{ACN})$ + ethyl formate $(\mathrm{EF})$. Experimental data this work: $(\diamond)$ Offline GC, $(\diamond)$ Online NMR; Prediction: (-) NRTL. 

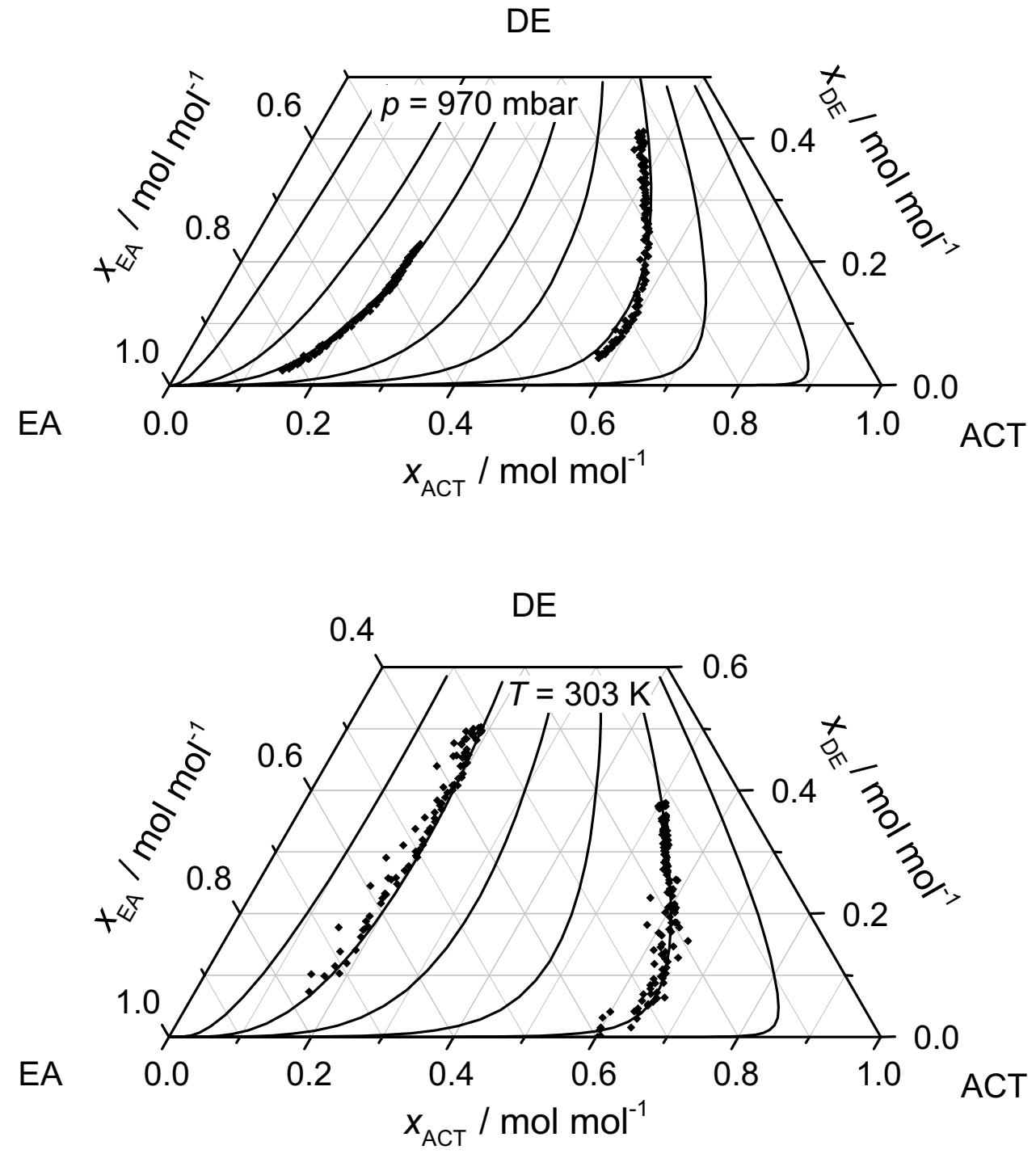

Figure 5: Results from measurements of two isobaric $(p=970$ mbar $)$ and two isothermal $(T=303 \mathrm{~K})$ residue curves in the system ethyl acetate $(\mathrm{EA})+$ acetone $(\mathrm{ACT})+$ diethyl ether (DE). Experimental data this work: $(\bullet)$ Online NMR; Prediction: $(-)$ NRTL. 

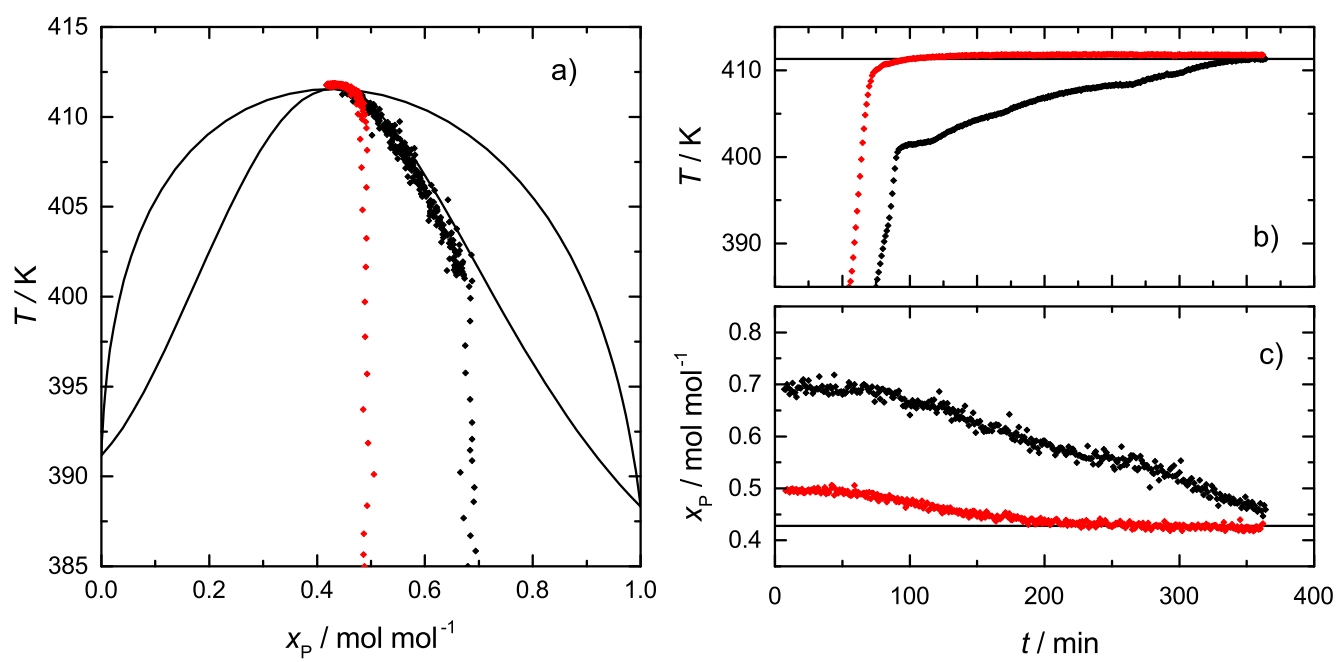

Figure 6: Results from isobaric ( $p=970$ mbar) distillation in the system acetic acid (AA) + pyridine $(\mathrm{P})$. a) Boiling curve as a function of mole fraction of pyridine in the liquid phase, b) Temperature as a function of the time, c) Mole fraction of pyridine in the liquid phase as a function of the time. Experimental data this work: Online NMR: $(\bullet)$ boiling curve, $(\bullet)$ azeotrope; Prediction: $(-)$ NRTL. 

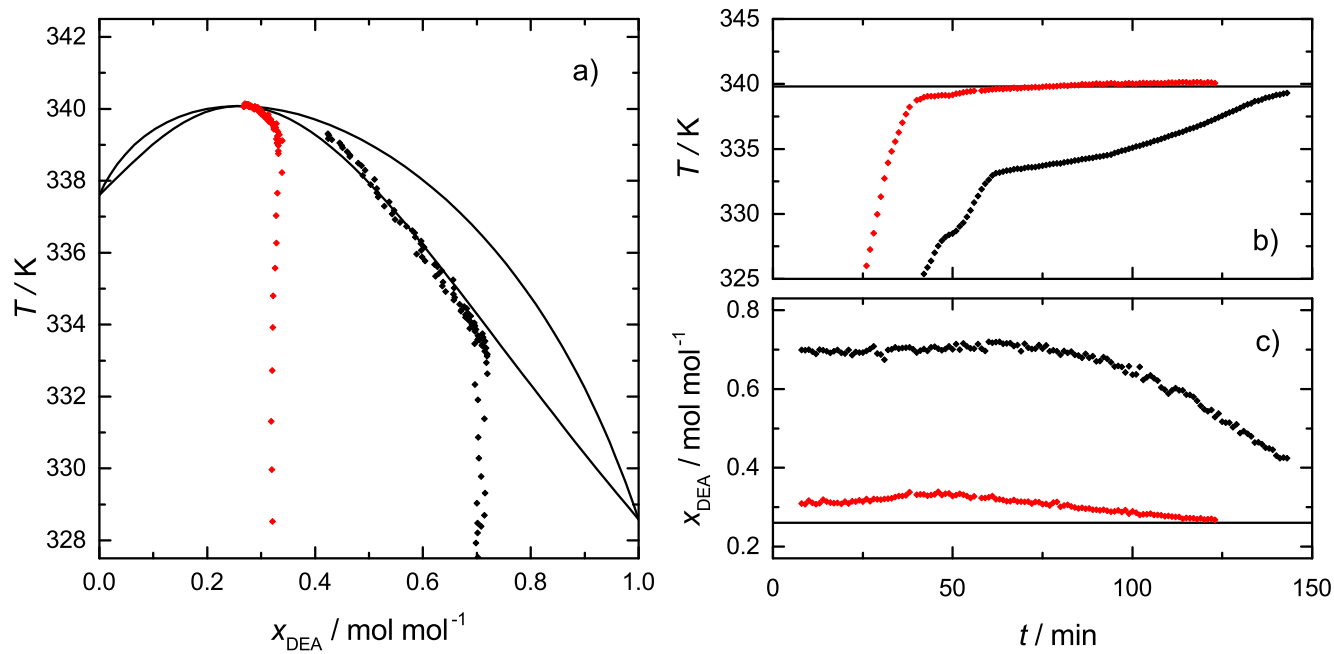

Figure 7: Results from isobaric ( $p=970$ mbar) distillation in the system methanol $(\mathrm{M})+$ diethylamine (DEA). a) Boiling curve as a function of mole fraction of diethylamine in the liquid phase, b) Temperature as a function of the time, c) Mole fraction of diethylamine in the liquid phase as a function of the time. Experimental data this work: Online NMR: $(\bullet)$ boiling curve, $(\diamond)$ azeotrope; Prediction: $(-)$ NRTL. 
Table 1: List of chemicals used for the investigations.

\begin{tabular}{llll}
\hline Chemical name & Source & Grade & Purity ${ }^{*}$ \\
\hline Acetic acid & Carl Roth & Rotipuran & $\geq 0.998 \mathrm{~g} / \mathrm{g}$ \\
Acetone & Merck & Uvasolv & $\geq 0.999 \mathrm{~g} / \mathrm{g}$ \\
Acetonitrile & Carl Roth & Rotisolv & $\geq 0.999 \mathrm{~g} / \mathrm{g}$ \\
Diethylamine & Acros Organics & ExtraPure & $\geq 0.990 \mathrm{~g} / \mathrm{g}$ \\
Diethyl ether & Sigma-Aldrich & ACS reagent & $\geq 0.995 \mathrm{~g} / \mathrm{g}$ \\
Dimethyl sulfoxide & Merck & Reagent Plus & $\geq 0.990 \mathrm{~g} / \mathrm{g}$ \\
Ethyl acetate & Sigma-Aldrich & ACS reagent & $\geq 0.995 \mathrm{~g} / \mathrm{g}$ \\
Ethyl formate & Sigma-Aldrich & ACS reagent & $\geq 0.970 \mathrm{~g} / \mathrm{g}$ \\
Methanol & Carl Roth & Anhydrous & $\geq 0.998 \mathrm{~g} / \mathrm{g}$ \\
Pyridine & Fisher & Analytical reagent grade & $\geq 0.999 \mathrm{~g} / \mathrm{g}$ \\
\hline
\end{tabular}

${ }^{*}$ specification of the supplier 
Table 2: High-boiling azeotrope in the systems $\mathrm{AA}+\mathrm{P}$ and $\mathrm{M}+\mathrm{DEA}$. Experimental NMR data from isobaric measurements at 970 mbar is compared to predictions by the NRTL model and literature data.

\begin{tabular}{lccc} 
& \multicolumn{3}{c}{ acetic acid + pyridine } \\
& $T / \mathrm{K}$ & $x_{\mathrm{P}} / \mathrm{mol} \mathrm{mol}^{-1}$ & $\mathrm{p} / \mathrm{mbar}$ \\
\hline NMR experiment & 411.8 & 0.421 & 970 \\
NRTL model & 411.0 & 0.428 & 970 \\
Swearingen and Ross [25] & 411.5 & 0.416 & 1013 \\
Zieborak et al. [26] & 411.3 & 0.422 & 1013 \\
Holmberg [27] & 411.2 & 0.410 & 1013 \\
\hline & & $\mathrm{methanol}+\mathrm{diethylamine}$ \\
& $T / \mathrm{K}$ & $x_{\mathrm{DEA}} / \mathrm{mol} \mathrm{mol}{ }^{-1}$ & $\mathrm{p} / \mathrm{mbar}$ \\
\hline NMR experiment & 340.1 & 0.268 & 970 \\
NRTL model & 339.8 & 0.260 & 970 \\
Aucejo et al. [28] & 339.8 & 0.245 & 1013 \\
Yang et al. [29] & 340.2 & 0.260 & 1013 \\
Nakanishi et al. [30] & 340.4 & 0.240 & 973 \\
\hline
\end{tabular}




\section{References}

[1] S. Kern, K. Meyer, S. Guhl, P. Gräßer, A. Paul, R. King, M. Maiwald, Online low-field NMR spectroscopy for process control of an industrial lithiation reaction - automated data analysis, Analytical and Bioanalytical Chemistry 410 (14) (2018) 3349-3360. doi:10.1007/s00216-0181020-z.

[2] Y. Matviychuk, E. von Harbou, D. J. Holland, An experimental validation of a bayesian model for quantification in NMR spectroscopy, Journal of Magnetic Resonance 285 (2017) 86 - 100. doi:10.1016/j.jmr.2017.10.009.

[3] R. Behrens, E. Kessler, K. Münnemann, H. Hasse, E. von Harbou, Monoalkylcarbonate formation in the system monoethanolaminewatercarbon dioxide, Fluid Phase Equilibria 486 (2019) 98 - 105. doi:10.1016/j.fluid.2018.12.031.

[4] A. Brächer, L. M. Kreusser, S. Qamar, A. Seidel-Morgenstern, E. von Harbou, Application of quantitative inline NMR spectroscopy for investigation of a fixed-bed chromatographic reactor process, Chemical Engineering Journal 336 (2018) 518 - 530. doi:10.1016/j.cej.2017.12.004.

[5] A. Brächer, R. Behrens, E. von Harbou, H. Hasse, Application of a new micro-reactor $1 \mathrm{H}$ NMR probe head for quantitative analysis of fast esterification reactions, Chemical Engineering Journal 306 (2016) 413 421. doi:10.1016/j.cej.2016.07.045. 
[6] O. Steinhof, G. Scherr, H. Hasse, Investigation of the reaction of 1,3dimethylurea with formaldehyde by quantitative on-line NMR spectroscopy: a model for the ureaformaldehyde system, Magnetic Resonance in Chemistry 54 (6) (2016) 457-476. doi:10.1002/mrc.4274.

[7] E. Danieli, J. Perlo, A. L. L. Duchateau, G. K. M. Verzijl, V. M. Litvinov, B. Blümich, F. Casanova, On-line monitoring of chemical reactions by using bench-top nuclear magnetic resonance spectroscopy, ChemPhysChem 15 (14) (2014) 3060-3066. doi:10.1002/cphc.201402049.

[8] V. Sans, L. Porwol, V. Dragone, L. Cronin, A self optimizing synthetic organic reactor system using real-time in-line NMR spectroscopy, Chem. Sci. 6 (2) (2015) 1258-1264. doi:10.1039/C4SC03075C.

[9] B. Gouilleux, B. Charrier, S. Akoka, F.-X. Felpin, M. Rodriguez-Zubiri, P. Giraudeau, Ultrafast 2D NMR on a benchtop spectrometer: Applications and perspectives, TrAC Trends in Analytical Chemistry 83A (2016) 65-75. doi:10.1016/j.trac.2016.01.014.

[10] J. M. Granda, L. Donina, V. Dragone, D.-L. Long, L. Cronin, Controlling an organic synthesis robot with machine learning to search for new reactivity, Nature 559 (7714) (2018) 377-381. doi:10.1038/s41586-0180307-8.

[11] M. Leutzsch, A. J. Sederman, L. F. Gladden, M. D. Mantle, In situ reaction monitoring in heterogeneous catalysts by a benchtop 
NMR spectrometer, Magnetic Resonance Imaging 56 (2019) 138-143. doi:10.1016/j.mri.2018.09.006.

[12] S. Kern, L. Wander, K. Meyer, S. Guhl, A. R. G. Mukkula, M. Holtkamp, M. Salge, C. Fleischer, N. Weber, R. King, S. Engell, A. Paul, M. P. Remelhe, M. Maiwald, Flexible automation with compact NMR spectroscopy for continuous production of pharmaceuticals, Analytical and Bioanalytical Chemistry 411 (2019) 3037-3046. doi:10.1007/s00216-019-01752-y.

[13] D. Kreyenschulte, E. Paciok, L. Regestein, B. Blümich, J. Büchs, Online monitoring of fermentation processes via non-invasive low-field NMR, Biotechnol. Bioeng. 112 (9) (2015) 1810-1821. doi:10.1002/bit.25599.

[14] K. Meyer, J.-P. Ruiken, M. Illner, A. Paul, D. Müller, E. Esche, G. Wozny, M. Maiwald, Process spectroscopy in microemulsions Setup and multi-spectral approach for reaction monitoring of a homogeneous hydroformylation process, Meas. Sci. Technol. 28 (2017) 1-11. doi:10.1088/1361-6501/aa54f3.

[15] S. S. Zalesskiy, E. Danieli, B. Blümich, V. P. Ananikov, Miniaturization of NMR systems: Desktop spectrometers, microcoil spectroscopy, and NMR on a chip for chemistry, biochemistry, and industry, Chem. Rev. 114 (11) (2014) 5641-5694. doi:10.1021/cr400063g.

[16] A. Friebel, A. Fröscher, K. Münnemann, E. von Harbou, H. Hasse, In situ measurement of liquid-liquid equilibria by medium field nu- 
clear magnetic resonance, Fluid Phase Equilibria 438 (2017) 44 - 52. doi:10.1016/j.fluid.2017.01.027.

[17] F. Dalitz, M. Cudaj, M. Maiwald, G. Guthausen, Process and reaction monitoring by low-field NMR spectroscopy, Progress in Nuclear Magnetic Resonance Spectroscopy 60 (2012) 52-70. doi:10.1016/j.pnmrs.2011.11.003.

[18] E. O. Fridjonsson, B. F. Graham, M. Akhfash, E. F. May, M. L. Johns, Optimized droplet sizing of water-in-crude oil emulsions using nuclear magnetic resonance, Energy Fuels 28 (3) (2014) 1756-1764. doi:10.1021/ef402117k.

[19] K. Meyer, S. Kern, N. Zientek, G. Guthausen, M. Maiwald, Process control with compact NMR, TrAC Trends in Analytical Chemistry 83A (2016) 39-52. doi:10.1016/j.trac.2016.03.016.

[20] J. Mitchell, L. F. Gladden, T. C. Chandrasekera, E. J. Fordham, Lowfield permanent magnets for industrial process and quality control, Progress in Nuclear Magnetic Resonance Spectroscopy 76 (2014) 1-60. doi:10.1016/j.pnmrs.2013.09.001.

[21] F. Dalitz, L. Kreckel, M. Maiwald, G. Guthausen, Quantitative MediumResolution NMR Spectroscopy Under Non-Equilibrium Conditions, Studied on the Example of an Esterification Reaction, Appl Magn Reson 45 (5) (2014) 411-425. doi:10.1007/s00723-014-0522-x.

[22] A. M. R. Hall, P. Dong, A. Codina, J. P. Lowe, U. Hintermair, Kinetics of asymmetric transfer hydrogenation, catalyst deactivation, 
and inhibition with noyori complexes as revealed by real-time highresolution flowNMR spectroscopy, ACS Catal. 9 (3) (2019) 2079-2090. doi:10.1021/acscatal.8b03530.

[23] M. Sawall, E. von Harbou, A. Moog, R. Behrens, H. Schröder, J. Simoneau, E. Steimers, K. Neymeyr, Multi-objective optimization for an automated and simultaneous phase and baseline correction of NMR spectral data, Journal of Magnetic Resonance 289 (2018) 132 - 141. doi:10.1016/j.jmr.2018.02.012.

[24] H. Renon, J. M. Prausnitz, Local compositions in thermodynamic excess functions for liquid mixtures, AIChe Journal 14 (1) (1968) 135-144.

[25] L. Swearingen, R. F. Ross, The system pyridine - acetic acid IV. boiling points, J. Phys. Chem. 39 (6) (1934) 821-828. doi:10.1021/j150366a010.

[26] K. Zieborak, D. Wyrzykowska-Stankiewicz, Bull. Acad. Pol. Sci. Ser. Sci. Chim. Geol. Geogr. 7 (4) (1959) 247-251.

[27] K. E. Holmberg, Physical properties of isotopic forms of azeotropic systems formed from acetic acid and some tertiary amines, Acta Chemica Scandinavica 13 (4) (1959) 717-721. doi:10.3891/acta.chem.scand.130717.

[28] A. Aucejo, S. Loras, R. Munoz, J. Wisniak, H. Segura, Phase equilibria and multiple azeotropy in the associating system methanol + diethylamine, Journal of Chemical and Engineering Data 42 (6) (1997) 1201-1207. doi:10.1021/je970115r. 
[29] C. Yang, Z. Qin, Y. Xu, H. Zeng, F. Sun, P. Zhang, Y. Feng, Isobaric vapor-liquid equilibrium for the binary systems of methanol, diethylamine, and $\mathrm{N}, \mathrm{N}$-diethylethanolamine at $\mathrm{p}=(60.0$ and 101.3$) \mathrm{kPa}, \mathrm{J}$. Chem. Eng. Data 58 (2) (2013) 482-487. doi:10.1021/je301219c.

[30] K. Nakanishi, H. Shirai, T. Minamiyama, Vapor-liquid equilibrium of binary systems containing alcohols. methanol with aliphatic amines, Journal of Chemical and Engineering Data 12 (4) (1967) 591-594. doi:10.1021/je60035a031. 